\title{
The hippocampus and contextual memory retrieval in Pavlovian conditioning
}

\author{
Stephen Maren *, William Holt \\ Department of Psychology and Neuroscience Program, University of Michigan, 525 E. University Avenue, Ann Arbor, MI 48109-1109, USA
}

Accepted 25 November 1999

\begin{abstract}
Several theories of hippocampal function have suggested a role for the hippocampus in contextual memory retrieval. However, these theories are based on studies using permanent pretraining lesions, which confound the role of the hippocampus in learning with its role in retrieval. We argue that an alternative methodology is required to assess the role of the hippocampus in memory retrieval processes. This alternative methodology involves temporary inactivation of the hippocampus in Pavlovian paradigms that lend themselves to an examination of retrieval. An example of this approach is considered in a Pavlovian fear-conditioning paradigm. We examined the influence of hippocampal inactivation on the context-specific expression of latent inhibition, a response decrement displayed when excitatory conditioning is preceded by non-reinforced presentations of a to-be-conditioned stimulus. Reversible inactivation of the dorsal hippocampus prior to retrieval testing eliminated the context-specific expression of latent inhibition. The nature of the hippocampal role in retrieval processes is discussed in the light of these data. (C) 2000 Elsevier Science B.V. All rights reserved.
\end{abstract}

Keywords: Hippocampus; Context; Muscimol; Retrieval; Fear; Interference; Latent inhibition; Freezing

\section{Introduction}

It has been recognized for decades that the expression of memory depends importantly on the context in which memory is retrieved [69]. For instance, it has been established that memory retrieval is facilitated to the degree that the context of memory retrieval is similar to the context of learning [68]. In many ways, our own day-to-day experiences reinforce this notion. For example, we all know that it is generally much easier to remember the name of a person that you meet while dining at a luxurious restaurant if you re-encounter that individual at the restaurant, as opposed to at the neighborhood grocery. Indeed, it is common to suffer memory lapses when people, places, or things are experienced 'out of context'. This process by which context facilitates memory retrieval is termed contextual

\footnotetext{
* Corresponding author. Tel.: +1-734-9366532; fax: +1-7347637480 .

E-mail address: maren@umich.edu (S. Maren)
}

memory retrieval. Contextual memory retrieval operates on many forms of learning and memory, including complex declarative memory in humans (as illustrated above) and simple forms of learning and memory in animals, including Pavlovian conditioning [4,5]. The present review will focus on the latter form of learning and memory.

Recent work in Pavlovian conditioning paradigms has demonstrated that environmental stimuli not only elicit learned behavioral responses (as in the sound of a metronome eliciting salivation), but also serve as the set of conditions under which certain associative contingencies are valid [68]. A process called occasion setting demonstrates this latter phenomenon $[6,54,61]$. For example, in a serial feature-positive discrimination (a form of positive occasion setting), a 'feature' stimulus, such as a tone, precedes a 'target' stimulus (the conditional stimulus or CS), such as a light, only when the target signals an unconditional stimulus (US), such as food or electric shock. Importantly, the feature is not presented on trials in which the US does not follow the target. Hence, the presence or absence of the feature- 
positive stimulus serves as a 'context' ${ }^{\text {' }}$ that controls behavior. In other words, the feature 'sets the occasion' for target-US trials [30]. Successful performance of such discriminations is obtained when the feature-positive stimulus retrieves the CS-US contingency. Thus, occasion setting illustrates the operation of contextual memory retrieval in Pavlovian conditioning paradigms.

In a typical occasion setting experiment, punctate stimuli, such as tones or lights, serve to retrieve the associations necessary for accurate performance. However, it has recently been appreciated that the training context (the static and diffuse environmental stimuli or background cues present during training) can also serve as an occasion setter in Pavlovian conditioning [6,24]. For example, Bouton [4] has documented many cases in which contextual stimuli select or retrieve the appropriate meanings of the punctate CSs that have previously occurred in that context, and thereby function similarly to the occasion setters described above. This function of context is readily apparent in Pavlovian paradigms in which a given CS has been programmed to have an ambiguous meaning. In these situations, contextual stimuli are used to disambiguate the meaning of the CS and gate appropriate behavioral performance to CSs. This function of context stands in contrast to its role as a CS, in which it can enter into compound associations or configurations with other stimuli as many associative

\section{Hirsh's Associative Model (Hippocampus-Independent)}

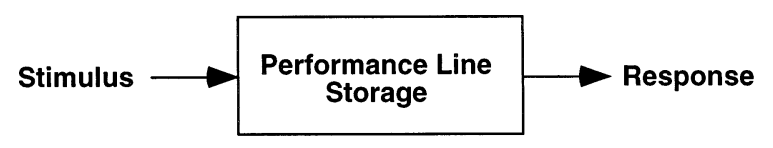

Hirsh's Retrieval Model
(Hippocampus-Dependent)

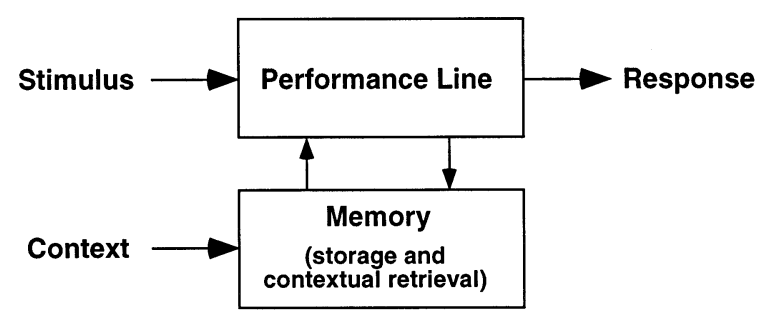

Fig. 1. Hirsh's memory models.

\footnotetext{
${ }^{1}$ In most cases, the term 'context' is used to describe the static and diffuse stimuli that characterize training and testing environments. Some investigators include variables such as time or interoceptive state in their description of context. And although punctate stimuli, such as brief tones or mild footshocks, are most often described as CSs or USs, some investigators also include these stimuli in their description of context.
}

theories would predict ${ }^{2}$. In either case, however, it is clear that contextual stimuli are importantly involved in regulating memory retrieval in Pavlovian conditioning paradigms.

In the last decade, there has been great interest in understanding the neural basis for contextual learning and memory processes. Much of this interested has been focused on the role of the hippocampal system (i.e. the hippocampus, dentate gyrus, subiculum, and entorhinal cortex) in contextual learning $[16,17,20,21,32,40,50,56,58,59,63,75]$. However, relatively few studies have explicitly examined the role of the hippocampus in contextual memory retrieval (for examples see Refs [18,20,34]). A recent synthesis of many lesion studies indicates that the hippocampus does mediate contextual processes in Pavlovian conditioning, however, the precise nature of the contextual processes is unclear [31]. In the present review, we take a modest step in attempting to delineate the role of the hippocampus in contextual retrieval during Pavlovian conditioning. We first review the historical literature concerning the role of the hippocampus in contextual memory retrieval. We then present experiments from our laboratory that characterize hippocampal function in retrieval processes by combining memory retrieval paradigms and temporary brain inactivation techniques. We conclude that the hippocampus permits contextual stimuli to retrieve the appropriate meaning of a CS when that CS has been trained to have more than one meaning.

\section{The hippocampus and contextual memory retrieval}

One of the first theories to implicate the hippocampus in contextual retrieval was proposed by Richard Hirsh in a seminal paper published in 1974 [28]. In this paper, Hirsh suggested that the hippocampus used contextual stimuli to label learned information in such a way that the information could later be retrieved in that context [28,29]. As noted above, Hirsh's notion of 'context' was broader than that used by other investigators examining Pavlovian contextual learning. He believed that contextual stimuli could consist of punctate signals (as in occasion setting), the static and diffuse cues associated with the training or testing environment, or the interoceptive states associated with food or

\footnotetext{
${ }^{2}$ Opinions have differed on how animals use contextual stimuli in a learning situation. Some theorists, such as Wagner [70,71], have proposed that contextual stimuli should be viewed as functionally similar to punctate stimuli, and thus should obey the same association principles. Others have suggested that contextual stimuli serve as modulators or 'occasion setters' to allow the disambiguation of stimuli with multiple meanings (e.g. Ref. [4]). Ultimately, it is likely that context serves both functions.
} 
water deprivation or drugs. In Hirsh's terms, contextual stimuli, "refer to but are not described within the information [the ambiguous stimulus and its meaning] to be retrieved" [28]. As shown in Fig. 1, he illustrated two potential models for information storage in the nervous system. In the 'associative' model, he imagined that information was stored along the 'performance line'. This is similar to most S-R theories of learning (e.g. Ref. [35]), and was not held to be dependent on hippocampal function. In addition, he elaborated a 'retrieval' model, in which memories were stored off the performance line and gained access to behavior through contextual retrieval processes. This contextual retrieval function was posited to require the hippocampus.

From Hirsh's viewpoint, intact rats used contextual encoding and retrieval processes to index or 'label' and recall multiple meanings of a particular set of stimuli according to its associated contextual signal. Rats without a functional hippocampus, on the other hand, do not have access to this indexing function, and instead are confined to using a single meaning for a given set of stimuli. Thus, when there is a discrepancy between a set of stimuli and its associated outcome (e.g. when a CS is suddenly not reinforced in extinction), adjustments in behavior in a hippocampal animal represent the summation of prior and present meanings of the stimulus as a purely S-R theory would predict. In contrast, intact rats can use contextual retrieval cues to rapidly adjust the meaning of a given set of stimuli as demanded by the environment; memory retrieval and behavioral performance occur when the ambiguous stimuli and their contextual retrieval cues co-occur. In other words, memory retrieval is directed by an 'AND gate' that detects the coincidence of a particular stimulus and the context in which it was learned.

For example, rats with hippocampal lesions typically do not have difficulty learning simple, non-conditional discriminations in which a US follows only one of two CSs $[20,34,62]$. When reinforcement contingencies are reversed however, rats with hippocampal lesions take roughly twice as many trials as intact rats to consistently perform according to the new contingencies (e.g. Refs [14,74]). Hirsh [28] explained this pattern of results by proposing that intact rats solve the discrimination reversal by applying contextual labels to the different sets of reinforcement contingencies. In contrast, rats with hippocampal lesions cannot use context to disambiguate the meaning of the CSs and therefore experience considerable interference between the two associations. In essence, hippocampal rats perform according to the sum of associative strengths acquired by the cues across all phases of training. As a consequence, Hirsh [28,29] and others (e.g. Ref. [57]) have suggested that hippocampal animals perform just as S-R theories of learning would predict.

Hirsh's theory is illustrated by the following experiment [29]. He trained rats on a T-maze in which one arm was baited with food and the other arm was baited with water. In the maze, he used the internal states of either hunger or thirst as discriminative stimuli for the correct response. Not surprisingly, intact rats learned this discrimination quickly, in about 50 trials. In contrast, rats that underwent neonatal irradiation of the hippocampus required nearly threetimes the number of training trials to reach a level of performance comparable to that of controls. In these rats, correct responses under one deprivation state corresponded nearly perfectly to incorrect responses under the other deprivation state. This indicates that rats with hippocampal lesions exhibit a high degree of perseveration towards one arm or the other arm of the T-maze without regard to the available contextual retrieval cues. In other words, hippocampal rats failed to exhibit contextual memory retrieval.

These data are consistent with the findings from a study by Ross and colleagues [62]. These investigators found that hippocampal aspirations in rats disrupted the acquisition of a serial feature-positive discrimination, but did not affect the acquisition of a simple, non-conditional discrimination. In these experiments, rats were trained using an appetitive conditioning procedure. A 5-s presentation of a light served as a feature stimulus that preceded a tone CS (the target) on trials on which the tone was followed by a food US. The light was not illuminated on trials in which the tone was not followed by the food US. The rats were simultaneously trained on a non-conditional discrimination in which an acoustic click (the CS+), but not a white noise (the $\mathrm{CS}-$ ), was followed by the US. After training in this procedure, intact rats exhibited two distinct CRs: a rearing CR to the light feature and a head jerk CR to the tone CS; after sufficient training, the head jerk CR was only exhibited on trials preceded by the light feature. Interestingly, rats with hippocampal lesions exhibited a normal rearing $\mathrm{CR}$ to the light feature and they learned to discriminate between the click and white noise. Nonetheless, they failed to limit their head jerk CRs to only those trials on which the tone was preceded by the light; in other words, they responded indiscriminately and at a high rate to the tone CS. As Ross and colleagues point out, this pattern of behavior suggests that there are at least two representations of the light feature: a representation of the light as predictor of the food US and a representation of the light that functions as a contextual retrieval cue (or 
occasion setter). Hippocampal damage appears to selectively disrupt the latter without affecting the former ${ }^{3}$.

Winocur and colleagues have also considered the role of the hippocampus in retrieval processes [74]. In their experiments, rats were trained in a simultaneous visual discrimination task in one of two contextually distinct runway apparatuses, and then retrained in the apparatus in which original learning occurred (no-shift condition) or in the other apparatus (shift condition). In each apparatus, the goal box was divided and entry to each compartment was gained through hinged doors on which visual stimuli (vertical or horizontal stripes) were placed. The rats were always reinforced for entering the door with horizontal stripes. However, one apparatus was always run under food deprivation and correct responses were reinforced with a food reward, whereas the other apparatus (which was considerably different in odor, texture, location, and lighting) was always run under water deprivation and correct responses were reinforced with a water reward. Rats with electrolytic hippocampal lesions performed at the same levels as control rats in learning the initial discrimination, and they also exhibited substantial savings when retrained in the same apparatus in the no-shift condition. However, rats with hippocampal lesions faired poorly in the shift condition, and required substantially more trials to achieve criterion performance. An additional experiment indicated that this deficit in performance was not simply as a result of distraction or external inhibition brought on by the novelty of the second test apparatus. Apparently, the absence of familiar contextual cues impaired retrieval of the cue-reward association in hippocampal rats.

Although Winocur and Olds [74] interpreted these data in terms of Hirsh's contextual retrieval theory, it is not necessarily clear why the contextual retrieval theory would predict this outcome. According to Hirsh, rats with hippocampal lesions should have been able to maintain accurate responding to the continuously reinforced visual cue despite the shift in context. That is, the meaning of the visual cues was never ambiguous, therefore contextual labeling was not required for performance of the task the horizontal stripes always predicted reward and the vertical stripes never predicted reward. An alternative account of these data is that the contextual stimuli present during training actually overshadowed the visual cues in rats with hippocampal lesions, thereby rendering highly context-specific responding [65].

More recently, Good and Honey [20,34] have examined the role of hippocampal function in contextual retrieval

\footnotetext{
${ }^{3}$ A recent study by Holland and colleagues [32] indicates that excitotoxic hippocampal lesions, which spare fibers of passage, do not affect the acquisition of serial feature-positive discriminations, but do impair the acquisition of serial feature-negative discriminations (see also Ref. [36]). Although these results are different from those of Ross and colleagues [62], they are nevertheless consistent with a role for the hippocampus in negative occasion setting.
}

using an appetitive Pavlovian procedure. In their first series of experiments, they found that electrolytic hippocampal lesions impaired the acquisition of a contextual conditional discrimination [20]. In this task, the rats received Pavlovian conditioning in two different contexts in which one of two CSs was followed by delivery of a food pellet. The CR consisted of an increased number of magazine entries during the $\mathrm{CS}+$ compared to the pre-CS baseline. Importantly, assignment of the cues as the $\mathrm{CS}+$ and $\mathrm{CS}-$ in each context was reversed such that the $\mathrm{CS}+$ in one context was the $\mathrm{CS}-$ in the alternate context. Hence, the contextual cues served to disambiguate the different meanings of each CS. Consistent with a role for the hippocampus in contextual retrieval, they found that electrolytic hippocampal lesions impaired the acquisition of this discrimination. Whereas intact rats readily learned to respond to each $\mathrm{CS}+$ in a context-specific manner, hippocampal rats did not differentiate between the cues and generally responded at a low rate to the cues. However, hippocampal lesions did not prevent the rats from learning a non-conditional contextual discrimination in which they used contextual cues to discriminate between an environment that had been paired with food USs and another environment that had not been paired with food USs. These results indicate that rats with hippocampal lesions can process contextual cues per se, but are severely impaired in using contextual cues to label and retrieve information about CSs in a context-appropriate manner.

In another series of experiments, Honey and Good [34] examined the influence of excitotoxic hippocampal lesions on latent inhibition, which is characterized by reduced conditional responding to a CS that has a previous history of non-reinforcement [46]. A hallmark of latent inhibition is that it is context-specific. That is, latent inhibition is attenuated if the contexts of CS preexposure and conditioning are different $[10,22,23,45]$, but can be 'renewed' if rats are tested in the context of CS preexposure following conditioning $[7,13,76]$. Honey and Good [34] used a within-subjects design and preexposed rats to a different CS in each of two different contexts. After the preexposure phase, they conditioned the rats by pairing both CSs with food reward in one of the two contexts. During conditioning, then, only one of the CSs had a history of non-reinforcement in the conditioning context. This CS should not have supported as much conditional responding as the CS that was preexposed in a different context insofar as latent inhibition is context-specific. This is exactly the pattern of results obtained in intact rats - conditional responding was acquired more slowly to the CS that had been preexposed in the conditioning context compared to the $\mathrm{CS}$ that had been preexposed outside of the conditioning context. Interestingly, hippocampal rats did not exhibit context-specific latent inhibition. They responded at a low rate to both of the CSs, suggesting that they exhibited latent inhibition to both CSs independent of where the CSs had been preexposed. 
Bouton has proposed that the context-specific expression of latent inhibition is the result of contextual retrieval processes [3,4]. Consistent with this view, Honey and Good [34] have argued that their results exemplify the role of the hippocampus in the retrieval processes illustrated by Hirsh [28,29]. By their view, rats with hippocampal lesions do not exhibit context-specific latent inhibition because they cannot use contextual labels to disambiguate the meaning of the CS, which has come to predict both non-reinforcement (a CS-'no event' association) and the US (a CS-US association) during different phases of training. Together with another experiment showing that hippocampal rats could acquire latent inhibition per se, Honey and Good [34] concluded that the hippocampus was required for the context-specific expression of latent inhibition.

\section{Permanent hippocampal lesions confound learning and retrieval}

The aforementioned studies provide support for a role for the hippocampus in contextual memory retrieval. However, these conclusions are tempered by the fact that all of these studies, with the exception of Ross and colleagues [62] (Experiment 2), used permanent pretraining lesions of the hippocampus. Unfortunately, this lesion methodology is problematic for interpreting the role of the hippocampus in rule-based contextual processes, because permanent pretraining lesions confound conditional learning and contextual retrieval. That is, when deficits in rats with permanent hippocampal lesions are obtained in these paradigms, one cannot be sure if the deficits are as a result of a failure to form conditional associations during training or a failure to retrieve these associations during testing. Therefore, the data linking the hippocampus to contextual retrieval are equivocal until it can be demonstrated that hippocampal disruption has a specific effect on retrieval processes, as opposed to an effect on the conditional learning.

Indeed, the timing of hippocampal lesions with respect to training can be a crucial determinant of the nature and extent of behavioral deficits in a variety of learning tasks. For example, we have recently demonstrated that pretraining and posttraining hippocampal lesions produce very different effects on contextual conditioning in a Pavlovian fear-conditioning task [50]. Specifically, we have found that rats with pretraining excitotoxic lesions of the dorsal hippocampal (DH) acquire contextual fear normally, that is, they exhibit high levels of freezing behavior in a context that has previously been associated with footshock [50]. However, rats that receive DH lesions shortly after fear conditioning exhibit robust impairments in contextual fear conditioning - they exhibit little freezing when returned to the conditioning context. This indicates that the timing of hippocampal lesions in this task is crucial. To explain this pattern of results, we have argued that rats can acquire contextual fear using at least two different strategies [51]. Normally, intact rats use a hippocampus-dependent strategy in which a configural representation of the context is formed and associated with the aversive US. This configural representation is therefore sensitive to posttraining disruptions of the hippocampus. However, rats with hippocampal lesions do not have access to the configural strategy, so they use an elemental strategy in which only a few of the most salient elements in the context are associated with the US.

The fact that intact rats might favor a configural representation of context is not surprising. Establishing configural representations or 'cognitive maps' appears to be the typical way mammals (particularly rodents) encode place representations, either for locating objects with in a particular place or to associate a place with a particular meaning. One could also imagine that a configural representation of contextual cues is more efficient than a series of elemental associations that hippocampal animals are believed to rely upon. The configural representations are information-rich in the sense that they not only contain all of the elemental information, but they also bind the elements together and allow for relational processing among the elements [11].

In addition to the timing of permanent hippocampal lesions, the methodology by which hippocampal lesions are made appears to be important. Thus, we have found that electrolytic and excitotoxic DH lesions produce different outcomes with respect to the acquisition of contextual fear conditioning [49,50]. As stated above, excitotoxic DH lesions made before training do not affect the acquisition of contextual fear conditioning. In contrast, pretraining electrolytic $\mathrm{DH}$ lesions produce modest impairments in contextual fear conditioning. Because electrolytic lesions produce damage to both DH neurons and axons passing through the hippocampus, these results reveal that extrahippocampal structures may be responsible for the contextual conditioning deficits often reported with electrolytic lesions or hippocampal aspirations. Moreover, Holland and colleagues [32] have recently found that while excitotoxic hippocampal lesions impair serial featurenegative discriminations, they do not impair the acquisition of serial feature-positive discriminations. These results contrast with those of Ross and colleagues [62] who found that hippocampal aspirations, which damage fibers of passage, impair feature-positive discriminations. Thus, the role for the hippocampus in contextual retrieval is further tempered by the fact that almost all of the aforementioned studies, with the exception of the studies by Holland [32] and Honey and 
Good [34], used lesion techniques that disrupt both hippocampal and extrahippocampal systems.

\section{Alternative methodologies}

The problems with the type and timing of permanent hippocampal lesions illustrate the need for alternative methodologies to explore the role of the hippocampus in contextual retrieval. Profitable methods for focusing on the role of the hippocampus in retrieval might include analysis of hippocampal function using either neuroimaging methods in humans or electrophysiological techniques in animals. Both of these methods have provided insight into the role of the hippocampus in memory retrieval $[8,17,64]$, although they do not allow inferences of causality.

Another alternative for studying hippocampal function in animals is to reversibly inactivate the hippocampus during restricted phases of behavioral training or testing (e.g. Refs [12,37]). Many reversible lesion studies have made use of pharmacological agents such as muscimol, a $\mathrm{GABA}_{\mathrm{A}}$ agonist that greatly potentiates inhibitory synaptic transmission in the brain $[27,37,41-44,48,55]$. Inactivation of discrete brain areas is obtained by intracranial microinfusion of small volumes $(0.1-0.5 \mu \mathrm{l})$ of the inactivating agent into the brain region of interest [52]. Because these agents have a relatively short half-life (less than $6 \mathrm{~h}$ ), animals can be subsequently tested in a functionally intact state within $24 \mathrm{~h}$ of inactivating a brain structure.

As noted above, a major problem with permanent lesion techniques is that they confound learning and retrieval processes. However, reversible lesions can circumvent this problem because they can be introduced during either the acquisition or retrieval phases of any particular behavioral task. Targeting particular phases of behavioral training or testing with reversible lesions is essential for achieving an understanding of the functional neuroanatomy of memory. With respect to examining the role for the hippocampus in contextual retrieval, this strategy would appear to be particularly amenable to an analysis of Pavlovian interference paradigms. For instance, Bouton [4] has described several Pavlovian interference paradigms (extinction, latent inhibition, counterconditioning, discrimination reversal learning, negative transfer, and learned irrelevance) in which contextual retrieval processes play an important role in behavioral performance. These retrieval processes can be isolated during extinction tests in which behavioral performance to a CS with a varied reinforcement history is measured in a target context. Bouton [4] has argued that the contextual cues present during testing reduce interference between the competing associative representations of a CS (for example, CS-'no event' and CS-US associations). In this way, contextual stimuli disambiguate the meaning of the CS and performance that is appropriate to the target context is obtained. Because these Pavlovian paradigms isolate a contextual retrieval process, they are ideally suited to studies involving reversible lesion techniques.

\section{Hippocampus and contextual retrieval in latent inhibition}

In the studies described below, we have taken advantage of this reversible lesion strategy to examine the role for hippocampal neurons in contextual retrieval processes operating in the latent inhibition paradigm. We have chosen to examine latent inhibition, because there are several studies to suggest an important role for the hippocampus in this form of learning $[1,2,9,25,34,38,39,53,60,66,67,77]$. Moreover, Honey and Good [34] have provided evidence for a role of the hippocampus in the contextual-specificity of latent inhibition. Nonetheless, Honey and Good [34] did not isolate retrieval processes per se, nor did their permanent hippocampal lesions have temporal specificity in their experiments. We have overcome both of these problems as described in the experiments below [33].

The first challenge in examining contextual retrieval is to define a behavioral design that isolates the retrieval process. Although latent inhibition is often characterized as a failure in associative or attentional processes that operate during conditioning, Bouton [4] has argued that latent inhibition represents a retrieval failure that results from interference between conflicting CS memories. That is, when CS preexposure and conditioning occur in the same context the CS-'no event' memory acquired during preexposure comes into direct competition with the CS-US association acquired during conditioning — the CS-'no event' memory acquired during preexposure proactively interferes with the excitatory CS association formed during conditioning. As a result, conditional responding to the CS is reduced. The fact that such interference regulates CR performance has been demonstrated by studies in which preexposure and conditioning occur in different contexts $[7,13,76]$. In these studies, CR performance is normal when extinction testing occurs in the conditioning context. Importantly, however, the $\mathrm{CR}$ is greatly diminished (or latently inhibited) when extinction testing occurs in the preexposure context. In this latter case, it appears as if the preexposure context retrieves the CS-'no event' memory, which consequently interferes with the expression of the CS-US memory acquired during conditioning. This illustrates that the expression of latent inhibition is context-specific. Although latent inhibition may also involve preexposure-related decrements in CS associability that operate during acquisition, these results reveal that contextual retrieval processes mediate 


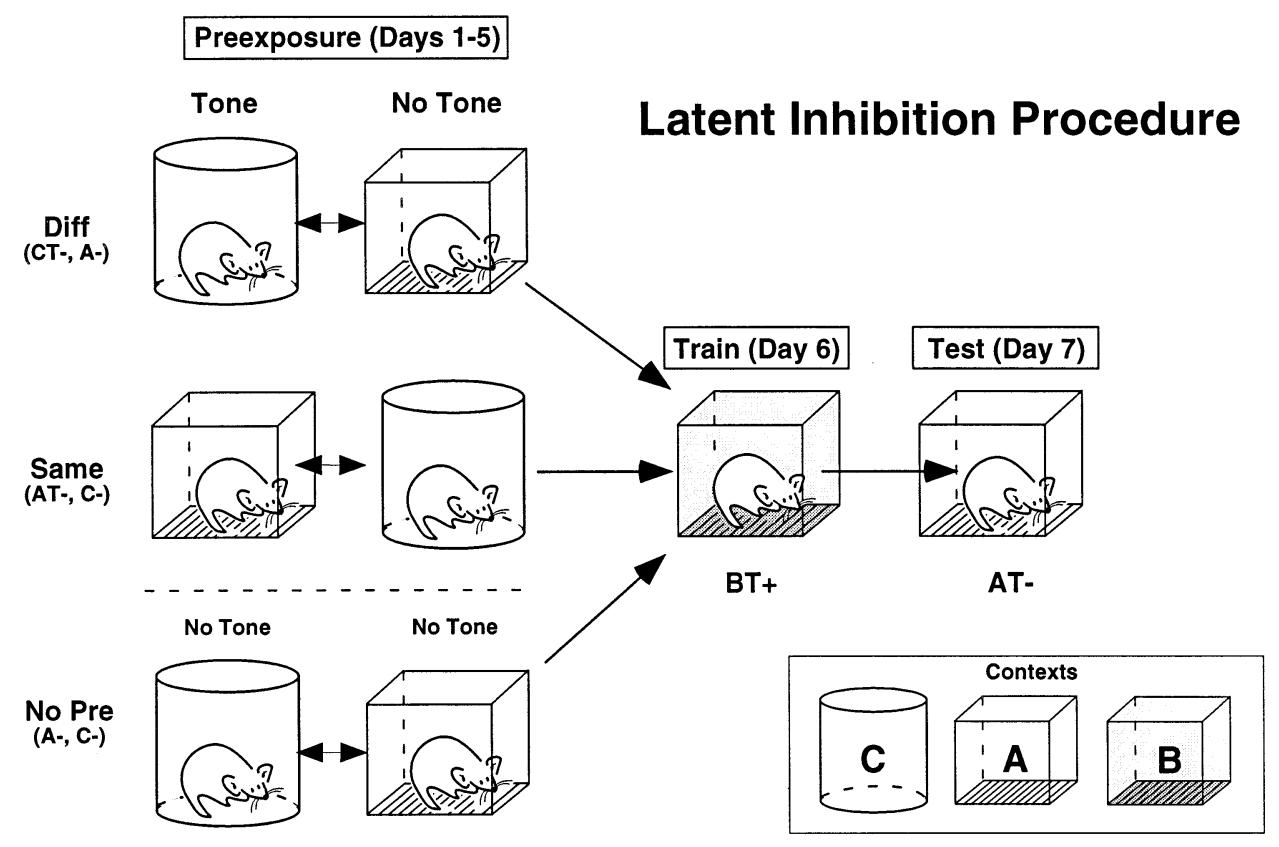

Fig. 2. Behavioral procedures for examining context-specific expression of latent inhibition in Pavlovian fear conditioning paradigm (A, B, and $\mathrm{C}=$ different contexts, $+=$ shock).

the expression of latent inhibition during retrieval testing.

Because this sort of design appears to isolate retrieval processes in latent inhibition, we chose to apply it to the fear-conditioning paradigm that we use in our laboratory. In Pavlovian fear conditioning, a neutral cue, such as a tone (the conditional stimulus or CS), will come to evoke a learned conditional fear response (CR) if it predicts the occurrence of an aversive event, such as an electric footshock (the unconditional stimulus or US). The fear CR consists of a number of autonomic and somatic responses, including fear-potentiated startle, hypoalgesia, enhanced cardiovascular output, and freezing [15].

In our first experiment, we examined whether the expression of latent inhibition in a conditional freezing paradigm was context-specific. As shown in Fig. 2, two groups of rats (SAME and DIFF) were preexposed to a tone stimulus in one of two experimental contexts (contexts A and C) on each of 5 days; a third group of rats (NO PRE) were not exposed to the tone. Rats in the SAME and DIFF groups received a total of 150 tone exposures. In all groups, total exposure to each context was equated. After the preexposure phase, all rats were placed in a third experimental context (context B) in which they received five conditioning trials in which the auditory stimulus used during the preexposure phase was paired with a footshock US. After 1 day following conditioning all of the rats were returned to context A for conditional fear testing (excitatory fear CRs readily transfer across test contexts). In this retrieval test, the rats were placed in context A and, after
$2 \mathrm{~min}$, the CS was presented continuously for $8 \mathrm{~min}$. Freezing during the CS served as the measure of conditional fear. Note that context $\mathrm{A}$ was the same as the context of preexposure for one group of rats (SAME), but was different from the context of preexposure for another group of rats (DIFF). Thus, CR performance only should be low in the SAME group, insofar as context A retrieves a memory for the preexposure phase that competes with the CS-US memory established during conditioning.

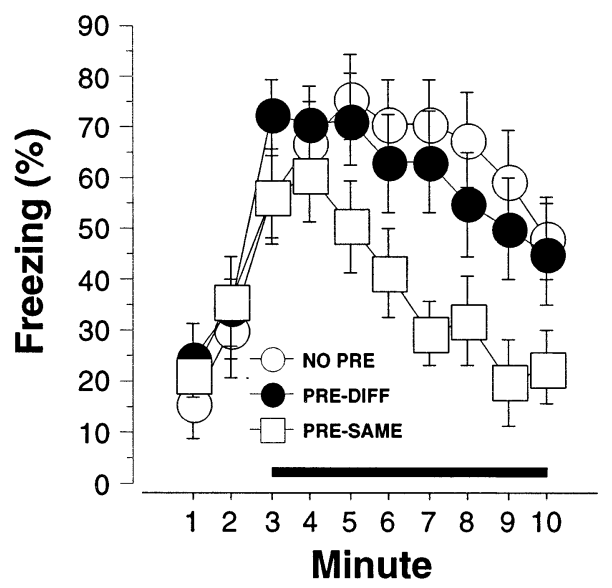

Fig. 3. Context-specific expression of latent inhibition in a conditional freezing paradigm. Mean $( \pm$ S.E.M.) percentage of freezing during an 8-min tone extinction test. Tone onset occurred at the start of the 3rd min of the test and is illustrated by the dark bar. Rats were tested in context A, which was either the same (SAME) or different (DIFF) from the context of tone preexposure; rats in the NO PRE group did not receive tone preexposure. 


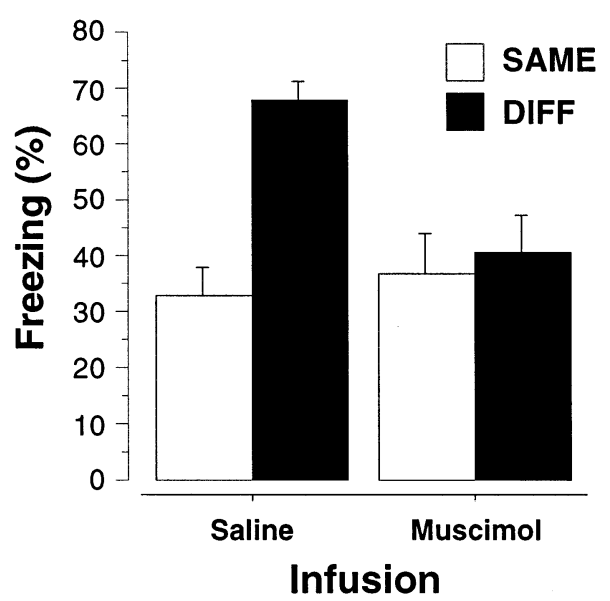

Fig. 4. Effects of hippocampal inactivation on the context-specific expression of latent inhibition. Mean $( \pm$ S.E.M.) percentage of freezing during a tone extinction test (collapsed across the 8-min test) in rats with hippocampal infusions of either muscimol or saline made prior to testing. Rats were tested in context A, which was either the same (SAME) or different (DIFF) from the context of tone preexposure.

Fig. 3 illustrates the outcome of the retrieval test. As expected, rats in the NO PRE grouped exhibited robust freezing to the tone CS. However, the SAME rats, which were preexposed to the tone CS in context A, exhibited a markedly attenuated freezing CR. This decrement in the freezing CR was not simply as a result of a prior history of CS preexposure, because rats in the DIFF group exhibited high levels of freezing that were indistinguishable from that of the non-preexposed controls. Consistent with earlier reports, these results indicate that the expression of latent inhibition is context-specific. This outcome reveals that retrieval processes operating at the time of testing are important in regulating $\mathrm{CR}$ performance in the latent inhibition paradigm.

The fact that retrieval processes can be isolated in the latent inhibition paradigm makes it an ideal preparation for examining the role of the hippocampus in contextual retrieval using reversible inactivation techniques. In our second experiment, we did just this and examined whether hippocampal inactivation would affect the context-specific expression of latent inhibition. A total of 1 week prior to behavioral testing, the rats were bilaterally implanted with guide cannula aimed at the DH. The behavioral procedures were identical to those described above, except that we omitted the nonpreexposed group. A total of $45 \mathrm{~min}$ prior to the retrieval test rats were infused with either muscimol (0.5 $\mu \mathrm{g}$ in $0.5 \mu \mathrm{l})$ or physiological saline $(0.5 \mu \mathrm{l})$ into the DH. As shown in Fig. 4, rats in the saline groups exhibited the same pattern of results as obtained in the first experiment. That is, rats tested in the same context as preexposure exhibited lower levels of freezing than rats tested in a context that was different from that of preexposure. The pattern of results in rats infused with muscimol was much different. Rats infused with muscimol exhibited low levels of freezing independent of whether they were tested in the same or different context as preexposure. Thus, inactivation of the DH eliminated the context-specific expression of latent inhibition. Importantly, however, the muscimol rats still exhibited latent inhibition - they showed low levels of freezing that were comparable to those in the SAME-saline rats. Thus, it appears as if the memory for the CS-'no event' association acquired during the preexposure came to dominate $\mathrm{CR}$ performance independent of the test context.

Although these results are consistent with a role for DH neurons in contextual retrieval, it may be the case that muscimol inactivation prevented the rats from using contextual information per se, without having a specific effect on contextual retrieval. To test this hypothesis, we examined the effect of DH inactivation on a simple, non-conditional contextual discrimination. For this task, rats received unsignaled footshock in one of two distinct contexts; no footshock was delivered in the alternate context. As in Experiment 2, the rats were infused with either saline or muscimol before conditional fear testing. Conditional freezing was measured in each context (one context test on each of 2 days). Rats receiving either saline or muscimol infusions into the DH exhibited a reliable contextual discrimination (data not shown). Both groups of rats exhibited high levels of freezing in the context in which unsignaled shock was delivered, and low levels of freezing in the safe context. This indicates that muscimol inactivation of the DH does not prevent all forms of context processing.

Together, these results provide a strong demonstration that neurons in the hippocampus are necessary for contextual retrieval in the latent inhibition paradigm. As shown in Fig. 5, we have conceptualized our data by suggesting that the hippocampus modulates the expression of latent inhibition via contextual retrieval of a CS-'no event' memory ${ }^{4}$. We imagine that the hippocampus allows context to regulate the expression of the CS-'no event' memory via an 'AND gate'. In the absence of a functional hippocampus, contextual retrieval cues cannot regulate expression of the CS-'no event' memory. As a result, expression of the CS-'no US' memory becomes context-independent and conditional responding to the CS is limited. Hence, rats with reversible hippocampal lesions behave as Hirsh would predict. Their performance is determined by the sum of their experiences with the CS, which in our experiments is heavily weighted towards a CS-'no event' memory as

\footnotetext{
${ }^{4}$ An alternative view is that the hippocampus is necessary to retrieve a configural representation of the context and CS, which is formed during the preexposure phase of training [45,63].
} 
a result of the comparatively large number of CS preexposure trials (150 preexposure trials vs five conditioning trials) the animals receive.

\section{Caveats and an explanatory model}

Although our data demonstrate a role for the hippocampus in contextual retrieval, there are several reports showing that rats with hippocampal lesions can perform the sorts of conditional operations thought to require this process. For example, rats with excitotoxic hippocampal lesions can acquire conditional discriminations, such as serial feature-positive discriminations $[32,36]$ or feature-ambiguous discriminations $[19,26]$. In considering these results, however, it is important to bear in mind that these studies made use of permanent pretraining lesions of the hippocampus. As discussed above, rats may adopt different learning strategies or engage compensatory neural systems to mediate conditional learning in the absence of the hippocampus. We have evidence to support this view in the contextual fear conditioning paradigm [50]. In addition, Rudy and Sutherland [63] have recently argued that the critical neural circuitry for encoding the sorts of stimulus configurations or conjunctions essential to conditional learning tasks is not in the hippocampus, but rather in
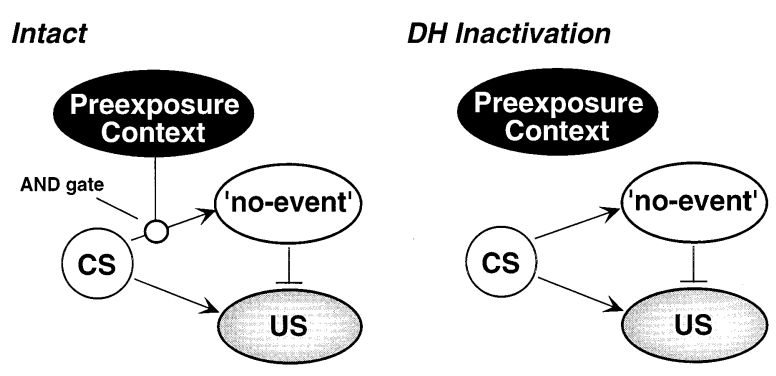

Fig. 5. A model of contextual retrieval in latent inhibition. We have elaborated Bouton's extinction model [5] to conceptualize contextual retrieval in latent inhibition. We imagine that a CS-'no event' association is acquired during preexposure and is only retrieved in the presence of the contextual stimuli that were associated with preexposure. Hence, an 'AND gate' is interposed between the CS and 'no event' representations and this gate allows contextual retrieval cues to regulate the expression of the CS-'no event' association. The expression of the excitatory CS-US association, which is acquired during conditioning, is context-independent. In the preexposure context, competition between the active CS-'no event' association and the CS-US association results in a suppression of the CS-US memory, favoring performance of the CS-'no event' memory. In this way, latent inhibition is only expressed in the context of preexposure. This allows for the expression of latent inhibition to be context-specific. We imagine that the hippocampus allows context to regulate the expression of the CS-'no event' memory via an 'AND gate'. In the absence of a functional hippocampus, contextual retrieval cues cannot regulate expression of the CS-'no event' memory. As a result, expression of the CS-'no US' memory becomes context-independent and conditional responding to the CS is limited. the cortex. They suggest that the hippocampus is involved in conditional learning insofar as it serves to enhance the salience of the stimulus conjunctions represented in cortex. While our latent inhibition data suggest that an intact hippocampus is required during testing to retrieve such stimulus conjunctions, it may be the case that rats with pretraining hippocampal lesions adopt a different neural system or retrieval strategy for this purpose.

Another experiment that has a direct bearing on the interpretation of our results was performed by Bouton and colleagues [73]. In this study, the investigators made electrolytic fimbria/fornix lesions prior to both conditioning and subsequent extinction of a Pavlovian bar-press suppression response (a fear conditioning procedure). Some of the rats received extinction training in the same context as conditioning, whereas other rats received extinction training in a different context. Following extinction, all rats were returned to the conditioning context and presented with the CS. Intact rats that had received extinction training in a context that was different from the conditioning context exhibited 'renewal' when they were tested for their fear of the $\mathrm{CS}$ in the conditioning context. In other words, they showed a high degree of response suppression (an index of fear) when the CS was tested in the conditioning context, despite the fact that response suppression had extinguished in the extinction context. This indicates that the ambiguous meaning of the CS (CS-US in the conditioning context and CS-'no US' in the extinction context) was disambiguated by a contextual retrieval process. In contrast with our latent inhibition results, however, they found that rats with fimbria/fornix lesions did not have a deficit in renewal. These data counter our conclusion that the hippocampus is necessary for the contextual retrieval processes that mediate context-specific expression of latent inhibition. However, it is important to note that Bouton and colleagues used fimbria/fornix lesions, which may have spared some aspects of hippocampal function [72], and that they made the lesions prior to behavioral training. In view of our results indicating spared contextual learning in rats with pretraining hippocampal lesions [50], it is reasonable to suggest that rats with hippocampal lesions may, under some conditions, use alternate strategies or neural systems for contextual retrieval.

The final issue that bears discussing is the role of the hippocampus in the acquisition versus the expression of latent inhibition. Because most studies have used permanent pretraining lesions of the hippocampus, there is a great deal of data indicating that hippocampal lesions impair the acquisition of latent inhibition $[1,2,25,34,38,39,53,66,67,77]$. In other words, rats with hippocampal lesions tend not to exhibit attenuate conditional responding after preexposure to the to-be-CS. However, as we have presently shown, hippocampal 
inactivation during retrieval testing does not impair the expression of latent inhibition per se - it merely eliminates the context-specificity of latent inhibition (see also Ref. [34]). In our hands, rats without a functional hippocampus are indeed more likely to exhibit reduced conditional responding than intact rats, at least in contexts that are different from those of preexposure.

We suggest that this pattern of results indicates that there are at least two distinct learning processes in the latent inhibition paradigm that require the hippocampus. We posit that the first process, which has been targeted by most permanent lesion studies, consists of the acquisition of a CS-'no event' memory during the CS preexposure phase of latent inhibition training ${ }^{5}$. We suggest that rats with hippocampal lesions do not encode a CS-'no event' representation during the CS preexposure phase of training. Hence, they do not learn that the CS has no consequence or that the CS is 'insignificant'. The failure to form such a representation might be indexed by the failure of rats with hippocampal lesions to decrement their orienting response to the CS during preexposure [38]. Although we believe that the hippocampus is required to encode CS-'no event' associations, we propose that this representation is not stored in the hippocampus. The second hippocampusdependent process we posit for latent inhibition is contextual memory retrieval $[4,28,68]$, which functions to retrieve the CS-'no event' association (presumed to reside outside of the hippocampus ${ }^{6}$ ) in the context of preexposure (see Fig. 5). As shown in Fig. 5, hippocampal inactivation at the time of contextual retrieval removes contextual-gating of the CS-'no event' memory and allows this memory to be expressed in a context-independent fashion (diminished conditional responding is observed in all test contexts).

This two-process model of latent inhibition accounts for impairments in the acquisition of latent inhibition after pretraining hippocampal lesions and the pre-

\footnotetext{
${ }^{5}$ There are competing theories as to the nature of the information acquired during CS preexposure. Wagner [70,71] holds that animals acquire context-CS associations that later block acquisition of the CS-US association. There is little evidence to support this view (e.g. Ref. [23]). Others hold that CS preexposure reduces the attention directed to the CS, and therefore reduces its associability [47]. We favor the view [4], that animals acquire a CS-'no event' association that later competes for performance with the CS-US association when preexposure and testing occur in the same context.

${ }^{6}$ We assume that the CS-'no event' association resides outside the hippocampus despite the fact that the expression of this association is context-specific. We make this assumption because hippocampal inactivation disrupts the contextual specificity of this memory, without affecting its expression per se. The idea that an association can be context-specific yet reside outside the hippocampus has also been suggested by studies indicating that context-US associations (which are by nature context-specific) are stored outside of the hippocampus [78].
}

served, yet context-independent, latent inhibition after hippocampal inactivation during retrieval testing. This model predicts that permanent hippocampal lesions made after CS preexposure, but before conditioning or retrieval testing, should eliminate the context specificity of latent inhibition without affecting the magnitude of latent inhibition per se. It also predicts that reversible inactivation of the hippocampus during CS preexposure should eliminate the acquisition of latent inhibition. Such experiments have yet to be performed.

\section{Conclusions and future directions}

The results of the latent inhibition experiments we have described provide strong evidence for hippocampal involvement in contextual memory retrieval. This conclusion is made much stronger by the fact that we used a reversible lesion technique together with a Pavlovian interference paradigm that isolates contextual retrieval processes. This strategy overcomes the problems associated with permanent, nonselective hippocampal lesions, which confound learning and retrieval and damage both hippocampal and extrahippocampal systems. Nonetheless, there are several important future directions for research in this area. First, as mentioned before, there are several Pavlovian interference paradigms (in addition to the latent inhibition paradigm) that require contextual retrieval for appropriate performance. Examining the impact of hippocampal inactivation during the retrieval phases of these paradigms is necessary to generalize the findings of our latent inhibition experiments. Moreover, our experiments rely upon a Pavlovian fear-conditioning paradigm in which conditional freezing as a behavioral measure. It is important, then, to extend our results to other Pavlovian responses, such as the appetitive CRs studied by Holland and colleagues [32]. Furthermore, it is necessary to determine if the hippocampus plays a general role in contextual retrieval, or if it is restricted to a subset of problems requiring this process, such as negative occasion setting [32].

In conclusion, context has been demonstrated to be a strong modulator of performance of learned behavior in animals and humans. Several attempts have been made to examine the relationships among contextual retrieval and the hippocampus using associative learning models. And while inroads have been made, permanent lesions prior to training confound the effects of the lesions on encoding with their effects on retrieval processes. Use of reversible lesions, such as those induced by muscimol microinfusion, together with behavioral procedures that specifically target various stages of memory (i.e. encoding, consolidation, or retrieval) may yield a sharper picture of the functional architecture of memory systems 


\section{Acknowledgements}

The research reported in this article was supported by grants from the NIMH (MH57360, MH57865) to SM.

\section{References}

[1] Ackil JE, Mellgren RL, Halgren C, Frommer GP. Effects of CS preexposures on avoidance learning in rats with hippocampal lesions. J Comp Physiol Psychol 1969;69:739-47.

[2] Baxter MG, Holland PC, Gallagher M. Disruption of decrements in conditioned stimulus processing by selective removal of hippocampal cholinergic input. J Neurosci 1997;17:5230-6.

[3] Bouton ME. Context and Retrieval in Extinction and in Other Examples of Interference in Simple Associative Learning, Current Topics in Animal Learning: Brain, Emotion, and Cognition. Hillsdale, NJ: Erlbaum, 1991:25-53.

[4] Bouton ME. Context, time, and memory retrieval in the interference paradigms of Pavlovian learning. Psychol Bull 1993;114:80-99.

[5] Bouton ME. Context, ambiguity, and classical conditioning. Curr Dir Psychol Sci 1994;3:49-53.

[6] Bouton ME, Swartzentruber D. Analysis of the associative and occasion-setting properties of contexts participating in a Pavlovian discrimination. J Exp Psychol: Anim Behav Process 1986;12:333-50.

[7] Bouton ME, Swartzentruber D. Slow reacquisition following extinction: context, encoding, and retrieval mechanisms. J Exp Psychol: Anim Behav Process 1989;15:43-53.

[8] Buckner RL, Raichle ME, Miezin FM, Petersen SE. Functional anatomic studies of memory retrieval for auditory words and visual pictures. J Neurosci 1996;16:6219-35.

[9] Buhusi CV, Gray JA, Schmajuk NA. Perplexing effects of hippocampal lesions on latent inhibition: a neural network solution. Behav Neurosci 1998;112:316-51.

[10] Channell S, Hall G. Contextual effects in latent inhibition with an appetitive conditioning procedure. Anim Learn Behav 1983;11:67-74.

[11] Cohen NJ, Eichenbaum H. Memory, Amnesia, and the Hippocampal System. Cambridge, MA: MIT Press, 1993:330.

[12] Dean P, Redgrave P, Lewis G. Locomotor activity of rats in open field after microinjection of procaine into superior colliculus or underlying reticular formation. Behav Brain Res 1982;5:175-87.

[13] Dexter WR, Merrill HK. Role of contextual discrimination in fear conditioning. J Comp Physiol Psychol 1969;69:677-81.

[14] Douglas RJ. The hippocampus and behavior. Psychol Bull 1967;67:416-22.

[15] Fanselow MS. What is conditioned fear? Trends Neurosci 1984;7:460-2.

[16] Frankland PW, Cestari V, Filipkowski RK, McDonald RJ, Silva AJ. The dorsal hippocampus is essential for context discrimination but not for contextual conditioning. Behav Neurosci 1998;112:863-74.

[17] Freeman JH Jr, Cuppernell C, Flannery K, Gabriel M. Contextspecific multi-site cingulate cortical, limbic thalamic, and hippocampal neuronal activity during concurrent discriminative approach and avoidance training in rabbits. $\mathbf{J}$ Neurosci 1996;16:1538-49.

[18] Freeman JH Jr, Weible A, Rossi J, Gabriel M. Lesions of the entorhinal cortex disrupt behavioral and neuronal responses to context change during extinction of discriminative avoidance behavior. Exp Brain Res 1997;115:445-57.
[19] Gallagher M, Holland PC. Preserved configural learning and spatial learning impairment in rats with hippocampal damage. Hippocampus 1992;2:81-8.

[20] Good M, Honey RC. Conditioning and contextual retrieval in hippocampal rats. Behav Neurosci 1991;105:499-509.

[21] Good M, de Hoz L, Morris RG. Contingent versus incidental context processing during conditioning: dissociation after excitotoxic hippocampal plus dentate gyrus lesions. Hippocampus 1998;8:147-59.

[22] Hall G, Minor H. A search for context-stimulus associations in latent inhibition. Q J Exp Psychol: Comp Physiol Psychol 1984;36B:145-69.

[23] Hall G, Honey RC. Contextual effects in conditioning, latent inhibition, and habituation: associative and retrieval functions of contextual cues. J Exp Psychol: Anim Behav Process 1989;15:232-41.

[24] Hall G, Mondragon E. Contextual control as occasion setting. In: Schmajuk NA, Holland PC, editors. Occasion Setting: Associative Learning and Cognition in Animals. Washington, DC: American Psychological Association, 1998:199-222.

[25] Han JS, Gallagher M, Holland P. Hippocampal lesions disrupt decrements but not increments in conditioned stimulus processing. J Neurosci 1995;15:7323-9.

[26] Han J-S, Gallagher M, Holland P. Hippocampal lesions enhance configural learning by reducing proactive interference. Hippocampus 1998;8:138-46.

[27] Helmstetter FJ, Bellgowan PS. Effects of muscimol applied to the basolateral amygdala on acquisition and expression of contextual fear conditioning in rats. Behav Neurosci 1994;108:10059.

[28] Hirsh R. The hippocampus and contextual retrieval of information from memory: a theory. Behav Biol 1974;12:421-44.

[29] Hirsh R. The hippocampus, conditional operations, and cognition. Physiol Psychol 1980;8:175-82.

[30] Holland PC. Temporal determinants of occasion setting in feature-positive discriminations. Anim Learn Behav 1986;14:11120.

[31] Holland PC, Bouton ME. Hippocampus and context in classical conditioning. Curr Opin Neurobiol 1999;9:195-202.

[32] Holland PC, Lamoureux JA, Han J-S, Gallagher M. Hippocampal lesions interfere with Pavlovian negative occasion setting. Hippocampus 1999;9:143-57.

[33] Holt W, Maren S. Muscimol inactivation of the dorsal hippocampus impairs contextual retrieval of fear memory. J Neurosci 1999;19:9054-62.

[34] Honey RC, Good M. Selective hippocampal lesions abolish the contextual specificity of latent inhibition and conditioning. Behav Neurosci 1993;107:23-33.

[35] Hull CL. Principles of Behavior. New York: Appleton-CenturyCrofts, 1943:422.

[36] Jarrard LE, Davidson TL. On the hippocampus and learned conditional responding: effects of aspiration versus ibotenate lesions. Hippocampus 1991;1:107-17.

[37] Jerusalinsky D, Quillfeldt JA, Walz R, Da Silva RC, Bueno e Silva M, Bianchin M, Schmitz P, Zanatta MS, Ruschel AC, Paczko N, et al. Effect of the infusion of the GABA-A receptor agonist, muscimol, on the role of the entorhinal cortex, amygdala, and hippocampus in memory processes. Behav Neural Biol 1994;61:132-8.

[38] Kaye H, Pearce JM. Hippocampal lesions attenuate latent inhibition and the decline of the orienting response in rats. Q J Exp Psychol: Comp Physiol Psychol 1987;39:107-25.

[39] Kaye H, Pearce JM. Hippocampal lesions attenuate latent inhibition of a CS and of a neutral stimulus. Psychobiology 1987;15:293-9.

[40] Kim JJ, Fanselow MS. Modality-specific retrograde amnesia of fear. Science 1992;256:675-7. 
[41] Knudsen EI, Knudsen PF. Contribution of the forebrain archistriatal gaze fields to auditory orienting behavior in the barn owl. Exp Brain Res 1996;108:23-32.

[42] Krupa DJ, Thompson JK, Thompson RF. Localization of a memory trace in the mammalian brain. Science 1993;260:98991.

[43] Krupa DJ, Weng J, Thompson RF. Inactivation of brainstem motor nuclei blocks expression but not acquisition of the rabbit's classically conditioned eyeblink response. Behav Neurosci 1996;110:219-27.

[44] Li CS, Mazzoni P, Andersen RA. Effect of reversible inactivation of macaque lateral intraparietal area on visual and memory saccades. J Neurophysiol 1999;81:1827-38.

[45] Lovibond PF, Preston GC, Mackintosh NJ. Context specificity of conditioning, extinction, and latent inhibition. J Exp Psychol: Anim Behav Process 1984;10:360-75.

[46] Lubow RE. Latent inhibition. Psychol Bull 1973;79:398-407.

[47] Mackintosh NJ. A theory of attention: variations in the associability of stimuli with reinforcement. Psychol Rev 1975;82:27698.

[48] Manning BH. A lateralized deficit in morphine antinociception after unilateral inactivation of the central amygdala. J Neurosci 1998;18:9453-70.

[49] Maren S, Fanselow MS. Electrolytic lesions of the fimbria/ fornix, dorsal hippocampus, or entorhinal cortex produce anterograde deficits in contextual fear conditioning in rats. Neurobiol Learn Mem 1997;67:142-9.

[50] Maren S, Aharonov G, Fanselow MS. Neurotoxic lesions of the dorsal hippocampus and Pavlovian fear conditioning in rats. Behav Brain Res 1997;88:261-74.

[51] Maren S, Anagnostaras SG, Fanselow MS. The startled seahorse: is the hippocampus necessary for contextual fear conditioning? Trends Cogn Sci 1998;2:39-42.

[52] Martin JH. Autoradiographic estimation of the extent of reversible inactivation produced by microinjection of lidocaine and muscimol in the rat. Neurosci Lett 1991;127:160-4.

[53] McFarland DJ, Kostas J, Drew WG. Dorsal hippocampal lesions: effects of preconditioning CS exposure on flavor aversion. Behav Neural Biol 1978;22:398-404.

[54] Miller RR, Oberling P. Analogies between occasion setting and Pavlovian conditioning. In: Schmajuk NA, Holland PC, editors. Occasion Setting: Associative Learning and Cognition in Animals. Washington, DC: American Psychological Association, 1998:3-35

[55] Muller J, Corodimas KP, Fridel Z, LeDoux JE. Functional inactivation of the lateral and basal nuclei of the amygdala by muscimol infusion prevents fear conditioning to an explicit conditioned stimulus and to contextual stimuli. Behav Neurosci 1997;111:683-91.

[56] Myers CE, Gluck MA. Context, conditioning, and hippocampal representation in animal learning. Behav Neurosci 1994;108:835-47.

[57] O'Keefe J, Nadel L. The Hippocampus as a Cognitive Map. Oxford: Oxford University Press, 1978:570.

[58] Penick S, Solomon PR. Hippocampus, context, and conditioning. Behav Neurosci 1991;105:611-7.

[59] Phillips RG, LeDoux JE. Differential contribution of amygdala and hippocampus to cued and contextual fear conditioning. Behav Neurosci 1992;106:274-85.

[60] Purves D, Bonardi C, Hall G. Enhancement of latent inhibition in rats with electrolytic lesions of the hippocampus. Behav Neurosci 1995;109:366-70.

[61] Ross RT, Holland PC. Conditioning of simultaneous and serial feature-positive discriminations. Anim Learn Behav 1981;9:293303.

[62] Ross RT, Orr WB, Holland PC, Berger TW. Hippocampectomy disrupts acquisition and retention of learned conditional responding. Behav Neurosci 1984;98:211-25.

[63] Rudy JW, Sutherland RJ. Configural association theory and the hippocampal formation: an appraisal and reconfiguration. Hippocampus 1995;5:375-89.

[64] Schacter DL, Wagner AD. Medial temporal lobe activations in fMRI and PET studies of episodic encoding and retrieval. Hippocampus 1999;9:7-24.

[65] Schmajuk NA. Psychological theories of hippocampal function. Physiol Psychol 1984;12:166-83.

[66] Schmajuk NA, Lam YW, Christiansen BA. Latent inhibition of the rat eyeblink response: effect of hippocampal aspiration lesions. Physiol Behav 1994;55:597-601.

[67] Solomon PR, Moore JW. Latent inhibition and stimulus generalization of the classically conditioned nictitating membrane response in rabbits (Oryctolagus cuniculus) following dorsal hippocampal ablation. J Comp Physiol Psychol 1975;89:1192203.

[68] Spear NE. Retrieval of memory in animals. Psychol Rev 1973;80:163-94.

[69] Tulving E, Thomson DM. Encoding specificity and retrieval processes in episodic memory. Psychol Rev 1973;80:352-73.

[70] Wagner AR. Expectancies and the priming of STM. In: Hulse $\mathrm{SH}$, Fowler H, Honig WK, editors. Cognitive Processes in Animal Behavior. Hillsdale, NJ: Erlbaum, 1978:177-209.

[71] Wagner AR. SOP: A model of automatic memory processing in animal behavior. In: Spear NE, Miller RR, editors. Information Processing in Animals: Memory Mechanisms. Hillsdale, NJ: Erlbaum, 1981:5-47.

[72] Whishaw IQ, Jarrard LE. Similarities vs differences in place learning and circadian activity in rats after fimbria-fornix section or ibotenate removal of hippocampal cells. Hippocampus 1995;5:595-604.

[73] Wilson A, Brooks DC, Bouton ME. The role of the rat hippocampal system in several effects of context in extinction. Behav Neurosci 1995; 109:828-36.

[74] Winocur G, Olds J. Effects of context manipulation on memory and reversal learning in rats with hippocampal lesions. J Comp Physiol Psychol 1978;92:312-21.

[75] Winocur G, Rawlins JN, Gray JA. The hippocampus and conditioning to contextual cues, Behav Neurosci 1987;101:617-25.

[76] Wright DC, Skala KD, Peuser KA. Latent inhibition from context-dependent retrieval of conflicting information. Bull Psychon Soc 1986;24:152-4.

[77] Yee BK, Feldon J, Rawlins JNP. Latent inhibition in rats is abolished by NMDA-induced neuronal loss in the retrohippocampal region, but this lesion effect can be prevented by systemic haloperidol treatment. Behav Neurosci 1995;109:22740.

[78] Young SL, Bohenek DL, Fanselow MS. NMDA processes mediate anterograde amnesia of contextual fear conditioning induced by hippocampal damage: immunization against amnesia by context preexposure. Behav Neurosci 1994;108:19-29. 